ISSN 0103-9954

\title{
ESPÉCIES EXÓTICAS INVASORAS ARBÓREAS NO PARQUE DA BARREIRINHA EM CURITIBA: REGISTRO E IMPLICAÇÕES
}

\author{
INVASIVE EXOTIC TREE SPECIES IN 'BARREIRINHA' PARK, CURITIBA: REGISTRATION AND \\ IMPLICATIONS
}

\author{
Erica Costa Mielke ${ }^{1}$ Raquel Rejane Bonato Negrelle ${ }^{2}$ Francine Lorena Cuquel $^{3}$ \\ Walquiria Pizatto Lima ${ }^{4}$
}

\begin{abstract}
RESUMO
A maioria das Unidades de Conservação (UCs) de Curitiba (PR, Brasil) está contaminada por espécies arbóreas exóticas invasoras (AEIs). Mas não existem estudos que evidenciem seu impacto e manejo apropriado. Os objetivos dessa pesquisa foram avaliar a participação das AEIs e as implicações e manejo da AEI de maior importância estrutural na composição florística-estrutural do Parque da Barreirinha, um remanescente de Floresta com Araucária. Foram avaliadas quatro unidades amostrais, com área total de $1600 \mathrm{~m}^{2}$ representando aproximadamente $4 \%$ da área total do maciço vegetal. Foram amostrados 354 indivíduos arbóreos, com DAP $\geq 15 \mathrm{~cm}$, e calculados, para cada espécie amostrada, os parâmetros de frequência relativa, densidade relativa, dominância relativa e valor de importância. Foram identificadas 29 famílias, 43 gêneros e 57 espécies, sendo três AEIs. A sustentabilidade e a integridade desta UC podem estar comprometidas devido à presença de Pittosporum undulatum Vent. (pau-incenso), espécie de maior valor de importância estrutural, alta densidade e frequência. Pelas características botânico-ecológicas do Pittosporum undulatum, a opção de remoção de indivíduos adultos deve ser considerada com muita cautela. Palavras-chave: Fitossociologia; Floresta Ombrófila Mista; Pittosporum undulatum; Unidade de Conservação.
\end{abstract}

\section{ABSTRACT}

The majority of the Conservation Units (CUs) of Curitiba (Paraná state, Brazil) are plagued by invasive alien tree species (IATs). However, no studies have been carried out to evaluate their impact or to determine the appropriate management techniques. The objective of this study is to evaluate the participation of IATs and the implications and management of the most significant species causing major structural change in the floristic-structural composition of 'Barreirinha' Park, a remnant of Araucaria Forest. Four plots were evaluated in a total area of $1600 \mathrm{~m}^{2}$ representing approximately $4 \%$ of the total area of the forest. A total of 354 individual trees were sampled with a diameter of $\geq 15 \mathrm{~cm}$, and for each species sampled, parameters were calculated of the relative frequency, relative density, relative dominance and the importance value. It was possible to identify 29 families, 43 genera and 57 species, three of which were IATs. The sustainability and integrity of this Conservation Unit can be threatened by the presence of Pittosporum undulatum (Sweet Pittosporum), the species with the greatest structural importance value, high density and frequency. Possible management strategies for the Pittosporum undulatum species are presented.

Keywords: Phytosociologicy; Mixed Ombrophilous Forest; Pittosporum undulatum Vent.; Conservation Units.

1 Engenheira Agrônoma, Dr $^{\mathrm{a}}$., Diretora do Departamento de Produção Vegetal da Prefeitura Municipal de Curitiba, Av. Senador Salgado Filho, 1050, CEP 80040-280, Curitiba (PR), Brasil. emielke@onda.com.br

2 Bióloga, Dr ${ }^{\mathrm{a}}$., Professora Associada do Departamento de Botânica, Universidade Federal do Paraná, Caixa Postal 19031, CEP 81530-990, Curitiba (PR), Brasil.negrelle@ufpr.br

3 Engenheira Agrônoma, Dr $^{\mathrm{a}}$., Professora Adjunta do Departamento de Fitotecnia, Universidade Federal do Paraná, Rua dos Funcionários, 1540, CEP 80035-050, Curitiba (PR), Brasil. francine@ufpr.br

4 Engenheira Florestal, M.Sc., Gerente de Parques e Bosques, Prefeitura Municipal de Curitiba, Av. Senador Salgado.Filho, 1050, CEP 80040-280, Curitiba (PR), Brasil. wlima@smma.curitiba.pr.gov.br 


\section{INTRODUÇÃO}

O ecossistema Floresta com Araucária ou Floresta Ombrófila Mista (FOM) tem sido considerado um dos mais importantes por abrigar espécies típicas, por apresentar atributos biológicos únicos em todo o planeta e igualmente, por ser exuberante em recursos madeiráveis e não madeiráveis. Não por coincidência, teve grande influência no desenvolvimento do Sul do Brasil a partir do início do século passado, gerando progresso e riqueza nessa região (AMBIENTE BRASIL, 2004). Por outro lado, o desenvolvimento desta região implicou na drástica redução deste ecossistema florestal, restando menos de $1 \%$ da sua área original de cobertura no Paraná (SANQUETTA, 2005).

Parte dos remanescentes de FOM está inserida em áreas urbanas sob forma de Unidades de Conservação (UCs). Elas são definidas pelo Sistema Nacional de Unidades de Conservação (SNUC, 2000) como espaço territorial com características naturais relevantes, legalmente instituído pelo Poder Público, com objetivo de conservação e limites definidos, sob regime especial de administração, ao qual se aplicam garantias adequadas de proteção. Elas representam uma das melhores estratégias de proteção do patrimônio natural (SESSEGOLO, 2006).

O Município de Curitiba, capital do Estado do Paraná, definiu suas UCs considerando o tamanho da área, representatividade fitogeográfica, conectividade, proteção de mananciais, diversidade de fitofisionomias, riqueza de espécies lenhosas e número de espécies raras e endêmicas, belezas naturais e prevenção quanto às enchentes (GILIOLI, 2006). Entretanto, em face da antropização oriunda dos processos de urbanização (KUHN e KLOTZ, 2006) observou-se nas UCs de Curitiba contaminação por AEIs (MIELKE et al., 2010). Essa contaminação interfere na dinâmica da floresta, principalmente no que diz respeito à regeneração e manutenção das espécies nativas, com redução da diversidade biológica e alteração da função e estrutura do respectivo ecossistema (SANQUETTA et al., 2005; GOODEN et al., 2009). Tão grave é esta contaminação que a presença de AEIs em ilhas e Unidades de Conservação (UCs) é considerada a primeira causa de perda de biodiversidade (CAMPOS e RODRIGUES, 2006). A problemática da contaminação por espécies exóticas invasoras em UCs já é conhecida, atingindo pelo menos 103
Unidades de Conservação brasileiras, espalhadas por 17 Estados e pelo Distrito Federal (GUIMARÃES, 2005).

Em Curitiba, 313 ha de remanescentes de FOM estão nas UCs, o que corresponde a apenas $5 \%$ de todo remanescente da cidade (CURITIBA, 2008). Ainda que de proporção minoritária, as UCs representam espaços únicos para a conservação ambiental.

O Parque da Barreirinha é um remanescente de FOM (Floresta Ombrófila Mista) e uma das mais antigas UCs de Curitiba. Criado em 1959 (CURITIBA, 2008), o parque apresenta contaminação por AEIs (MIELKE et al., 2010). No entanto, não existem estudos que evidenciam o impacto e manejo apropriado das AEIs nas áreas que ocupam. Portanto, os objetivos dessa pesquisa foram avaliar a participação das AEIs, e sua implicação, além do manejo da AEI de maior importância estrutural na composição florísticaestrutural neste Parque.

\section{MATERIAL E MÉTODO}

\section{Local de estudo}

O estudo foi realizado no Parque da Barreirinha $\left(49^{\circ} 21^{\prime} \mathrm{S}\right.$ e $49^{\circ} 15^{\prime} \mathrm{O}, 934$ m s.n.m), localizado em Curitiba, Paraná. O clima da região é classificado, segundo Köppen, como $\mathrm{Cfb}$, que apresenta características de clima subtropical mesotérmico, superúmido, com verões frescos e geadas severas, demasiadamente frequentes (média de 5 geadas/ano), sem estação seca. A temperatura média anual é de $16,5^{\circ} \mathrm{C}$, com média do mês mais quente e mais frio de $20,1^{\circ} \mathrm{C}$ e $12,8^{\circ} \mathrm{C}$, respectivamente. $\mathrm{O}$ mês mais chuvoso é janeiro e o menos é agosto (MAACK, 1981). Os solos predominantes nas áreas não hidromórficas são os Latossolos, Cambissolos e Organossolos e nas áreas hidromórficas são os Gleissolos (ITCG, 2010).

O Parque da Barreirinha apresenta área total de $132.014 \mathrm{~m}^{2}$, sendo a $42.920,68 \mathrm{~m}^{2}$ de remanescente de FOM, além de áreas com vegetação graminoide, áreas arbóreas isoladas, lagos, construções e trilhas. Estas últimas permitem ao visitante percorrer todo o parque, não havendo qualquer restrição de visitação dentro de seus limites. Está situado ao norte de Curitiba, distante cerca de oito km do seu centro. 


\section{Estabelecimento das unidades amostrais e método de coleta}

Para a realização da pesquisa foi avaliada uma área de $1600 \mathrm{~m}^{2}$ (aproximadamente $4 \%$ da área do remanescente da FOM), dividida em quatro unidades amostrais $(20 \times 20 \mathrm{~m})$. A seleção das unidades amostrais ocorreu por meio de estudo preliminar, observando-se gradiente visual dos indivíduos adultos da principal árvore exótica invasora, Pittosporum undulatum, distribuídas de modo a representar a vegetação arbórea do Parque, a saber: Unidade amostral 1: próxima ao acesso principal do Parque e a imóveis particulares. Presença de indivíduos adultos e regeneração abundante de Pittosporum undulatum; Unidade amostral 2: presença de indivíduos adultos e regeneração moderada de Pittosporum undulatum; Unidade amostral 3: apenas regeneração de Pittosporum undulatum, em menor proporção que nas unidades 1 e 2; Unidade amostral 4: pouca regeneração de Pittosporum undulatum e presença de indivíduos de Araucaria angustifolia.

Dentro de cada unidade amostral todas as árvores com diâmetro acima do peito (DAP) $\geq$ $15 \mathrm{~cm}$, incluindo as árvores mortas em pé, foram incluídas no estudo. Para cada indivíduo amostrado foi mensurado o DAP. A identificação botânica foi feita por botânicos do Museu Botânico Municipal de Curitiba, com a utilização de chaves analíticas e consultas a especialistas. Os indivíduos arbóreos foram classificados de acordo com o sistema APG II (ANGIOSPERM PHYLOGENY GROUP II, 2003) e a nomenclatura das espécies foi verificada em Missouri Botanical Garden (2010). Estas foram classificadas de acordo com o grupo ecológico, entre não pioneiras e pioneiras.

\section{Parâmetros analisados}

A similaridade entre as áreas foi calculada pelo Índice de Similaridade Florística de Sorensen (Js) que é dado em porcentagem (MATTEUCCI e COLMA, 1982) e a diversidade avaliada pelo Índice de Shannon (H') (MAGURRAN, 1988) cuja significância foi calculada por meio do teste do Qui - quadrado $\left(\mathrm{X}^{2}\right)$. Dominância, frequência, densidade e valor de importância das espécies arbóreas foram determinados de acordo com Mueller-Dombois e Ellenberg (1974).

O impacto das plantas exóticas sobre as nativas foi calculado segundo o Índice de Impacto
Ambiental de Exóticas (IIAE), efetuado a partir do cálculo dos coeficientes do impacto ambiental (REASER et al., 2007). Este índice varia de -1 a 1 , sendo que -1 significa que a área não possui plantas nativas e 1 que a área não possui plantas exóticas.

\section{RESULTADOS E DISCUSSÃO}

Foram identificados 354 indivíduos, de 29 famílias, 43 gêneros e 57 espécies (Tabela 1). Dentre estas, três identificadas como AEIs (Pittosporum undulatum Vent. - pau-incenso, Hovenia dulcis Thunb. - uva-japão e Ligustrum japonicum Thunb alfeneiro), correspondendo a 5,88\% do número total de indivíduos avaliados. Estas espécies estavam restritas a apenas duas unidades amostrais.

Para o componente amostral relativo aos indivíduos vivos detectaram-se valores de diversidade (H') variando entre 2,30 e 2,88. Nas parcelas com presença de árvores exóticas registraram-se menores valores absolutos de $\mathrm{H}^{\prime}$, sendo estes significativamente distintos quando comparados entre unidades amostrais, havendo em uma delas presença de AEIs. Considerando cada uma das unidades amostrais separadamente, observou-se baixa similaridade florística entre elas (Js $<50 \%$ ) (Tabela 2).

Nas unidades amostrais 1 e 2 houve ocorrência de árvore exótica invasora. Sob o contexto estrutural, observou-se que o Pittosporum undulatum representava o segundo $(49,85)$ e o terceiro $(29,57)$ maiores valores de importância, respectivamente, especialmente devido a sua alta dominância e distribuição homogênea. Nestas unidades amostrais observou-se também menor número indivíduos representativos das espécies nativas, bem como menor número total de espécies. Além disto, registrou-se IIAE inferior a 0,80 indicando que estas áreas teriam aproximadamente uma ocupação de $20 \%$ de vegetação exótica invasora (Tabela 3).

Em relação ao grupo ecológico observouse a porcentagem de espécies não pioneiras, sempre superior àquelas consideradas pioneiras (Figura 1).

Um dos critérios para que uma área seja configurada como UC, em Curitiba, é a qualidade de sua vegetação (GILIOLI, 2006). Nesta perspectiva, o Parque da Barreirinha deveria estar preservado quanto a sua integridade biológica. No entanto, verificou-se nesse local uma cobertura vegetal heterogênea, com baixa diversidade, apesar 
TABELA 1: Espécies arbóreas identificadas por amostragem na Floresta Ombrófila Mista no Parque da Barreirinha, Curitiba, PR.

TABLE 1: Species sampled in Forest with Araucaria, in 'Barreirinha' Park, Curitiba, PR state, Brazil.

\begin{tabular}{|c|c|c|}
\hline Família & Nome vulgar & Nome científico \\
\hline Anacardiaceae & Bugreiro & Lithraea brasiliensis March. \\
\hline Anacardiaceae & Aroeira & Schinus terebinthifolius Raddi \\
\hline Annonaceae & Ariticum & Rollinia rugulosa (Schltdl.) \\
\hline Aquifoliaceae & Erva-mate & Ilex paraguariensis A. St. Hil. \\
\hline Araucariaceae & Araucária & Araucaria angustifolia (Bert.) O. Ktze \\
\hline Asteraceae & Sem nome & Critoniopsis quinqueflora (Less.) H. Rob. \\
\hline Asteraceae & Cambará & Piptocarpha axillaris (Less.) Baker \\
\hline Asteraceae & Vassourão-branco & Piptocarpha angustifolia Dusén ex Malme \\
\hline Bignoniaceae & Ipê-mirim & Cybistax antisyphilitica (Mart.) Mart. \\
\hline Bignoniaceae & Caroba & Jacaranda puberula Cham. \\
\hline Canellaceae & Pimenteira & Cinamodendron dinisii (Schwacke) Occhioni \\
\hline Cannabaceae & Juvevê & Celtis pubescens Spreng. \\
\hline Celastraceae & Coração-de-bugre & Maytenus evonymoides Reissek \\
\hline Celastraceae & Sem nome & Maytenus sp. \\
\hline Clethraceae & Canjuja & Clethra scabra Pers. \\
\hline Erythroxylaceae & Cocão & Erythroxylum deciduum A. St-Hill. \\
\hline Euphrobiaceae & Leiteiro & Sapium glandulatum (Vell.) Pax \\
\hline Fabaceae & Falso-barbatimão & Cassia leptophylla Vogel \\
\hline Fabaceae & Ingá-feijão & Inga marginata Willd. \\
\hline Fabaceae & Marmeleiro & Dalbergia brasiliensis Vogel \\
\hline Lauraceae & Canela-sebo & Nectandra megapotamica (Spreng.) Mez \\
\hline Lauraceae & Canela-fedida & Ocotea bicolor Vattimo \\
\hline Lauraceae & Sassafraz-do-campo & Ocotea elegans $\mathrm{Mez}$ \\
\hline Lauraceae & Canela-sassafrás & Ocotea odorifera Rohwer \\
\hline Lauraceae & Canela-sebo & Ocotea puberula (Rich.) Nees \\
\hline Lauraceae & Sem nome & Ocotea SP \\
\hline Lauraceae & Sem nome & Ocotea $\mathrm{sp} 1$ \\
\hline Malvaceae & Açoita-cavalo & Luehea divaricata Mart. \\
\hline Meliaceae & Canjerana & Cabralea canjerana (Vell.) Mart. \\
\hline Myrsinaceae & Capororoquinha & Myrsine coriacea R. Br. ex Roem. \& Schult. \\
\hline Myrsinaceae & Sem nome & Myrsine sp. \\
\hline Myrsinaceae & Capororoca & Rapanea umbellata (Mart Ex DC) Mez \\
\hline Myrtaceae & Guavirova & Campomanesia xanthocarpa O. Berg \\
\hline Myrtaceae & Guamirim & Eugenia capitulifera $\mathrm{O}$. Berg \\
\hline Myrtaceae & Pitanga & Eugenia uniflora $\mathrm{L}$. \\
\hline Myrtaceae & Guamirim & Myrcia rostrata DC. \\
\hline Myrtaceae & Craveiro & Pimenta pseudocaryophyllus (Gomes) Landrum \\
\hline Oleaceae & Alfeneiro & Ligustrum japonicum Thunb. \\
\hline Pittosporaceae & Pau-incenso & Pittosporum undulatum Vent. \\
\hline Rhamnaceae & Uva-japão & Hovenia dulcis Thunb. \\
\hline Rhamnaceae & Sem nome & Rhamnus sp. \\
\hline
\end{tabular}


TABELA 1: Continuação ...

TABLE 1: Continued ...

\begin{tabular}{lcl}
\hline \multicolumn{1}{c}{ Família } & Nome vulgar & \multicolumn{1}{c}{ Nome científico } \\
\hline Rhamnaceae & Pau-pombo & Rhamnus sphaerosperma Sw. \\
Rosaceae & Pessegueiro-bravo & Prunus brasiliensis Dietrich \\
Rubiaceae & Sem nome & Psychotria sp. \\
Rutaceae & Mamica & Zanthoxylum rhoifolium Lam. \\
Salicaceae & Guaçatunga & Casearia decandra Jacq. \\
Salicaceae & Cambroé & Casearia lasiophylla Eichler \\
Salicaceae & Guaçatunga & Casearia sylvestris Sw. \\
Salicaceae & Sucará & Xylosma pseudosalzmanii Sleumer \\
Sapindaceae & Vacum & Allophylus edulis (A. St.-Hil., Cambess. \& A. Juss.) Radlk. \\
Sapindaceae & Cuvatã & Cupania vernalis Cambess. \\
Sapindaceae & Miguel-pintado & Matayba elaeagnoides Radlk. \\
Solanaceae & Quina & Solanum pseudoquina A. St.-Hill. \\
Solanaceae & Sem nome & Solanum sp. \\
Solanaceae & Sem nome & Solanum sp1 \\
Styracaceae & Carne-de-vaca & Styrax leprosus Hook \& Arn. \\
Symplocaceae & Sem nome & Symplocos sp. \\
\hline
\end{tabular}

TABELA 2: Índice de similaridade de Sorensen (Js) e índice de diversidade de Shannon (H’), entre as unidades amostrais, Parque da Barreirinha, Curitiba - PR.

TABLE 2: Sorensen similitary index $(\mathrm{Js})$ and Shannon diversity index $\left(\mathrm{H}^{\prime}\right)$ between sampling units, Barreirinha Park, Curitiba, PR state, Brazil.

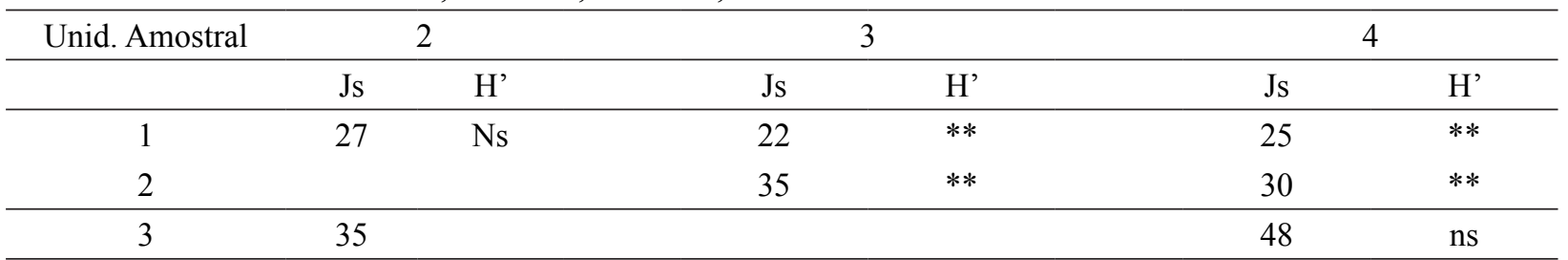

Em que: **diferença significativa ao nível de $1 \%\left(X^{2}\right)$

de esta apresentar espécies ocorrentes da FOM, comparativamente a outras áreas no Estado do Paraná (REGINATO et al., 2008; ROSEIRA, 1990; KOZERA et al., 2006). Neste cenário, registrouse a presença de espécies exóticas invasoras, destacando-se o Pittosporum undulatum (pauincenso) por seu alto valor de importância estrutural $(49,85$ e 29,57$)$. A presença desta espécie pode influenciar na estrutura e dinâmica da mata nativa (GUARIGUATA e OSTERTAG, 2001), uma vez que altera processos ecológicos, como: ciclagem de nutrientes, taxas de decomposição, cadeias tróficas, processos evolutivos, polinização, estrutura, perdas de biodiversidade e valor estético de paisagem (ZILLER, 2000). A ocorrência de espécies invasoras no Parque da Barreirinha não é um evento isolado, dado que já foi observado em outros levantamentos florísticos realizados em remanescentes de FOM, sendo três deles em UCs de Curitiba. Nestes registrou-se a presença de pelo menos quatro AEIs: Hovenia dulcis e Ligustrum sp, Morus nigra e Pittosporum undulatum (BARDAL et al., 2004; CORDEIRO, 2005; ROSEIRA, 1990; KOZERA et al., 2006; IURK et al., 2009; REGINATO et al., 2008; ROTTA, 1977, MIELKE et al., 2010).

A descaracterização da vegetação original pela presença de AEIs ao longo do tempo foi fato reconhecido por inúmeros autores (PIMENTEL et al., 2008; CERVI et al., 1987; GLEADOW e NARAYAN, 2007). De acordo com Schaff et al. (2006), a predominância da uva-japão (Hovenia dulcis) em São João do Trinfo - PR em relação à 
TABELA 3: Composição florística-estrutural das áreas amostrais no Parque da Barreirinha, Curitiba - PR. $\mathrm{S}=$ riqueza, $\mathrm{H}^{\prime}=$ índice de diversidade de Shannon, $\sigma=$ variância, $\mathrm{Da}=$ densidade absoluta, $\mathrm{DoA}=$ dominância absoluta, IIAE = índice de impacto ambiental de exóticas, $\mathrm{DR}=$ densidade relativa, Dor $=$ dominância relativa, $\mathrm{VI}=$ valor de importância.

TABLE 3: Floristic data and structural sampling areas in 'Barreirinha' Park, Curitiba, PR state, Brazil. $\mathrm{S}=$ species richness, $\mathrm{H}^{\prime}=$ Shannon diversity index, $\sigma=$ variance, $\mathrm{Da}=$ absolute density, $\mathrm{Do} A=$ absolute dominance, $\mathrm{IIAE}=$ index of environmental impact of exotic, $\mathrm{DR}=$ relative density, Dor $=$ relative dominance, $\mathrm{VI}=$ importance value.

\begin{tabular}{|c|c|c|c|c|c|c|c|c|c|c|c|c|c|}
\hline \multirow{2}{*}{ Unid. Amostral } & \multicolumn{5}{|c|}{ GERAL } & \multicolumn{5}{|c|}{ EXÓTICAS INVASORAS } & \multicolumn{3}{|c|}{ MORTAS } \\
\hline & $\mathrm{S}$ & $\mathrm{H}^{\prime}$ & $\sigma$ & DA & DoA & $\mathrm{S}$ & IIAE & DR & Dor & VI & $\mathrm{S}$ & Dor & VI \\
\hline 1 & 15 & 2,30 & 0,015 & 1425 & 1,06 & 3 & 0,57 & 17,75 & 29,76 & 64,18 & 5 & 3,49 & 24,8 \\
\hline 2 & 21 & 2,41 & 0,016 & 2000 & 1,16 & 2 & 0,75 & 13,58 & 9,86 & 36,26 & 1 & 0,83 & 4,63 \\
\hline 3 & 30 & 2,84 & 0,010 & 2925 & 1,50 & 0 & 0 & 0 & 0 & 0 & 3 & 1,24 & 4,59 \\
\hline 4 & 23 & 2,88 & 0,007 & 2125 & 1,67 & 0 & 0 & 0 & 0 & 0 & 2 & 2,00 & 8,07 \\
\hline
\end{tabular}

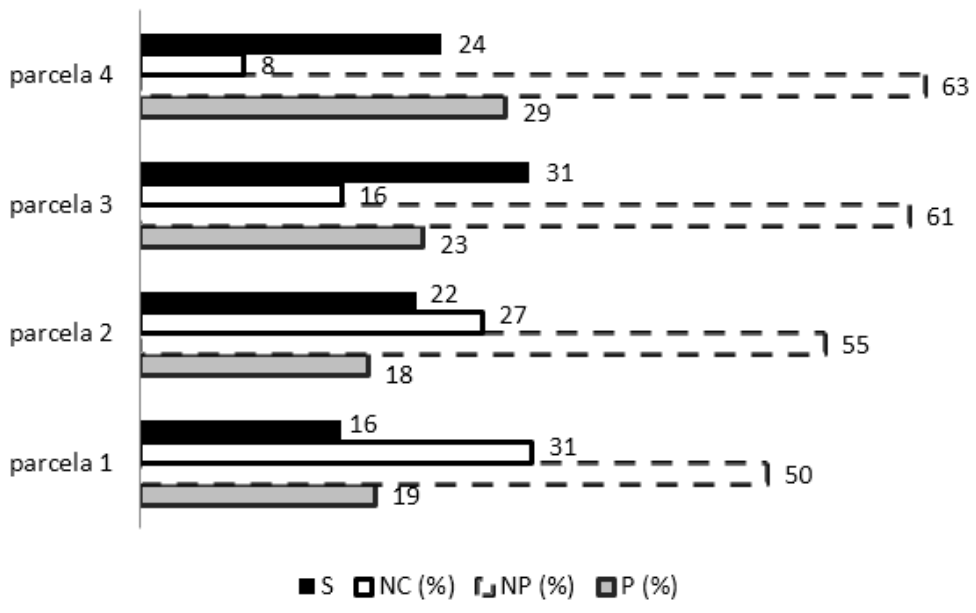

FIGURA 1: Status sucessional do Parque da Barreirinha. $\mathrm{S}=$ riqueza, $\mathrm{NC}=$ espécie não citada quanto ao status sucessional (\%), NP = espécie não pioneira (\%) e $\mathrm{P}=$ espécie pioneira (\%), Curitiba PR.

FIGURE 1: Sucessional status of 'Barreirinha' Park. $\mathrm{S}=$ richness species, $\mathrm{NC}=$ species not listed (\%), $\mathrm{NP}=$ non-pioneer species (\%) and $\mathrm{P}=$ pioneer species (\%), Curitiba, PR state, Brazil.

mata nativa foi percebida de um ano para outro. Em outro estudo, a vegetação exótica invasora (Eryobotrya japonica Lindl., Impatiens walleriana Hook. F., Hovenia dulcis e Tecoma stans (L.) Kunth) em Farroupilha - RS, ocupava 14\% do remanescente florestal nativo e se localizava próxima às trilhas e à circulação de pessoas (MAGGIONI e LAROCCA, 2009). A alteração estrutural evidenciada no presente estudo pode ser um dos indicadores que confirmam o processo de infestação e já pode, no Parque da Barreirinha, estar impactando o equilíbrio ambiental. Fato este que deve se replicar em todas as áreas de remanescentes naturais onde há incidência de espécies exóticas invasoras.

Outro fator indicativo da alteração de estrutura em função da presença de árvores invasoras é a baixa diversidade $\left(<2,9\right.$ nat.ind $\left.^{-1}\right)$. Em áreas remanescentes da FOM, onde a presença de AEI não foi significativa, a ponto de interferir na dinâmica dessa floresta, os índices de diversidade resultaram em valores significativamente mais elevados $\left(3,43\right.$ a 8,11 nat.ind $\left.{ }^{-1}\right)$ (RONDON NETO et al., 2002; NEGRELLE e LEUCHTENBERGER, 2001; REGINATO et al., 2008; NEGRELLE e 
SILVA, 1992). Deste modo, é possível supor que a área em estudo pode estar de fato comprometida ou estar minimamente em processo de degradação pela presença das árvores exóticas invasoras.

Os resultados obtidos para o Índice de Impacto de Ambiental de Exóticas (IIAE) $(0,57 \mathrm{e}$ $0,75)$ também apontam no sentido da interferência das espécies exóticas invasoras na integridade do remanescente florestal estudado, indicando, segundo Reaser et al. (2007), a necessidade de intervenção e manejo, pois valores de IIAE abaixo de 0,80 representariam que cerca de $20 \%$ da área estaria ocupada por vegetação exótica.

O estabelecimento de espécies nativas pode estar sendo prejudicado por haver interferências desde o processo de germinação dessas espécies devido ao efeito alelopático do pau-incenso (GLEADOW e ASHTON, 1981, GOODLAND e HEALEY, 1997). Portanto, o monitoramento das espécies exóticas invasoras, envolvendo avaliação da regeneração, banco de plântulas e banco de sementes é necessário para antever a condição estrutural e de sustentabilidade da floresta, e propiciar medidas cabíveis de controle.

A origem da contaminação biológica do Parque da Barreirinha não está bem esclarecida. Uma das hipóteses é de que a contaminação biológica tenha dado início pela ação humana (antropização). Em conversa com funcionários antigos, há relatos de que próximo à área da unidade amostral 1 , na qual houve maior incidência de Pittosporum undulatum existia uma área de agricultura que foi abandonada. Talvez pela maior fertilidade e por ser uma área limpa, ou seja, livre de vegetação, sementes de Pittosporum undulatum tenham encontrado ali o local propício para germinação. Contribuiu ainda para contaminação biológica, a ação direta dos indivíduos de Pittosporum undulatum plantados na área de estacionamento do Parque, o que certamente promoveu a dispersão desta espécie naquela área. A escolha da espécie foi devido às suas características ornamentais (BIONDI e PEDROSA-MACEDO, 2008) e a dispersão aparentemente foi favorecida pela abertura de trilhas onde ocorre maior circulação de pessoas e de clareiras (IURK et al., 2009). No estudo em pauta parece que a antropização tem sido a maior responsável pela alta incidência de Pittosporum undulatum que atualmente compõe a paisagem do Parque da Barreirinha

Pelos dados obtidos, não foi possível afirmar categoricamente e sim apenas intuir, que o Pittosporum undulatum apresentava-se como o fator limitante para o desenvolvimento da mata nativa. Entretanto, considerando que esta espécie apresenta forte competitividade por polinizadores, comprovado potencial alelopático e elevada produção de propágulos (37.500 sementes/ind.) em conjunto com eficiente dispersão ornitocórica (GOODLAND e HEALEY, 1997) e, além disto, elevada capacidade de crescimento e rebrota e de crescimento favorecido frente à abertura de clareiras (ROSE, 1997), esta espécie deve ser alvo de monitoramento e controle contínuo, especialmente em relação à sua regeneração na UC em questão, pois é evidente sua capacidade de dispersão e estabelecimento.

As características do Pittosporum undulatum aliadas às ações antrópicas, citadas anteriormente, podem ser restritivas às práticas usuais de somente remoção dos seus indivíduos adultos, uma vez que pouco se conhece sobre a dinâmica do banco de sementes e banco de plântulas desta espécie. Além disto, considerando a capacidade de adaptação do Pittosporum undulatum como uma espécie pioneira (MULLETT, 1999) e o recrutamento de plântulas ocorrer sob o dossel da floresta, processo este que se intensifica pela abertura de clareiras (BINGGELI e GOODLAND, 1997), a ação de remoção pode dinamizar a distribuição de propágulos ou reforçar a ocorrência desta espécie na área em estudo. $\mathrm{O}$ controle das plântulas de Pittosporum undulatum poderia eficazmente ser feito por métodos químicos (GOODLAND e HEALEY, 1997). Contudo, a Portaria 14, de 26 de maio de 2010 do IBAMA, que autoriza o uso de produtos químicos para controle de vegetação exótica invasora em áreas de floresta nativa, em caráter emergencial, inclui diversas plantas assim classificadas, exceto o Pittosporum undulatum. Desta forma, o controle desta espécie por meio de controle químico em UCs no meio urbano, torna-se inviável por falta de amparo legal.

Com vistas às ações de controle ou erradicação das AEIs em UCs é absolutamente fundamental avaliar, considerar e conscientizar a comunidade que vive no entorno e que usufrui dessas áreas. Se não houver convencimento por parte destes grupos, estes se tornarão motivadores de resistência a qualquer interferência que verse sobre a erradicação de qualquer espécie invasora (OLIVEIRA e PEREIRA, 2010) atrasando, onerando e até impedindo a conclusão da erradicação das AEIs. É sabido que a educação ambiental pode ser um importante instrumento para melhorar a 
percepção da população quanto às causas ambientais (ARAÚJO et al., 2010) não apenas para que ela entenda a importância das ações de erradicação em UCs, mas também para que ela não seja responsável pelas introduções intencionais ou não das AEIs.

Considerando os resultados obtidos nesta pesquisa, a conjuntura das UCs e a opinião pública da sociedade sobre erradicação de AEIs são possíveis duas estratégias de manejo do Pittosporum undulatum:

1. Remoção dos indivíduos adultos em conjunto com campanhas permanentes de educação ambiental da população sobre os problemas das plantas exóticas invasoras em áreas de mata nativa.

2. Manutenção dos indivíduos adultos de Pittosporum undulatum nas áreas de mata nativa, sem abertura de clareiras e com podas anuais permanentes das inflorescências, a fim de impedir a produção de sementes.

Em ambas as estratégias o banco de plântulas deve ser controlado permanentemente através de arranquio.

\section{CONCLUSÕES}

O Parque da Barreirinha apresenta contaminação pelas seguintes árvores exóticas invasoras: Pittosporum undulatum, Hovenia dulcis e Ligustrum lucidum. Destacando-se a primeira pelo alto valor de importância estrutural em função do grande número de indivíduos e elevada frequência. Desta forma, a sustentabilidade e a integridade da mata nativa do Parque da Barreirinha podem estar comprometidas. Devido às características botânicoecológicas do Pittosporum undulatum, a remoção de indivíduos adultos deve ser considerada com muita cautela. Estudos futuros sobre a dinâmica dessa espécie serão fundamentais para o estabelecimento de metas visando a ações de manejo e controle eficazes, assim como campanhas de educação ambiental.

\section{REFERÊNCIAS BIBLIOGRÁFICAS}

APG II. an update of the Angiosperm Phylogeny Group classification for the orders and families of flowering plants: APG II. Botanical Journal of the Linnean Society, San Francisco, CA, v. 141, n. 4, p. 399-436, 2003.

ARAÚJO, J. de L. O., ARAÚJO, A. C. de; ARAÚJO, A. C. de. Percepção ambiental dos residentes do bairro Presidente Médici em Campina GrandePB, no tocante à arborização local. REVSBAU, Piracicaba, v. 5, n. 2, p. 67-81, 2010.

AMBIENTE BRASIL. Projeto pinhão. "Floresta Ombrófila Mista". Disponível em: $<$ (http://pinho. floresta.ufpr.br/ pinhao/floresta.htm) $>$ Acesso em: 28 de março de 2009.

BARDDAL, M. L. et al. Caracterização florística e fitossociológica de um trecho sazonalmente inundável de floresta aluvial, em Araucária, Pr. Ciência Florestal, Santa Maria, v. 14, n. 2, p. 37-50, 2004.

BINGGeli, P.; GOODLAND, T. Pittosporum undulatum Vent. (Pittosporaceae). Disponível em: $\quad<$ (http://members.multimania.co.uk/ WoodyPlantEcology/docs/web-sp15.htm)> Acesso em: 08 de maio de 2011.

BIONDI, D.; PEDROSA-MACEDO, J. H. Plantas invasoras encontradas na área urbana de Curitiba (PR). Floresta, Curitiba, v. 38, n. 1, p. 129-144, 2008.

CAMPOS, J. B.; RODRIGUES, L. S. R. Eliminação de Espécies Exóticas nas Unidades de Conservação Estadual do Paraná. In: CAMPOS, J.B.; TOSSUliNO, M.G.P.; MÜLLER, C.R.C. (Org.). Unidades de Conservação: ações para valorização da biodiversidade. Curitiba: Instituto Ambiental do Paraná. 2006, p. 120-125.

CERVI, A. C. et al. Levantamento do estrato arbóreo do capão da Educação Física da Universidade Federal do Paraná Curitiba - Paraná - Brasil. Estudos de Biologia, Curitiba, n. 17, p. $49-61$, ago, 1987.

CORDEIRO, J. Levantamento florístico de caracterização fitossociológica de remanescente de Floresta Ombrófila mista em Guarapuava, PR. 2005. 144 f. Tese (Doutorado) - Universidade Federal do Paraná, Curitiba, 2005.

CURITIBA. Plano municipal de controle ambiental e desenvolvimento sustentável - III Versão, Curitiba, 2008. Disponível em: $<$ (www. curitiba.pr.gov.br) $>$ Acesso em: 02 de agosto de 2010.

GILIOLI, L. N. Parques e Bosques em Curitiba no Paraná. 2006. 135 f. Dissertação (Mestrado em Programação dos Ambientes Urbanos Sustentáveis) - Pontifícia Universidade Católica do Paraná, Curitiba, 2006.

GLEADOW, R. M.; ASHTON, D. H. Invasion by Pittosporum undulatum of the forests of central Victoria. I: Invasion patterns and plant morphology. Australian Journal of Botany, Collingwood, v. 29, 
p. $705-20,1981$.

GLEADOW, R. M.; NARAYAN, I. Temperature thresholds for germination and survival of Pittosporum undulatum: Implications for management by fire. Acta Oecologica, n. 31, p. 131-157, 2006.

GOODEN, B. et al. Invasion and management of a woody plant, Lantana camara L., alters vegetation diversity wet sclerophyll forest in southeastern Australia. Forest Ecology and Management, n. 257, p. 960-967, 2009.

GOODLAND, T.; HEALEY, J. R. The control of the Australian tree Pittosporum undulatum in the Blue Mountains of Jamaica. Bangor: School of Agricultural and Forest Science. University of Wales, 1997.

GUARIGUATA, M. R.; OSTERTAG. R. Neotropical secondary forest succession: changes in structural and functional characteristics. Forest Ecology and Management, v. 148, p. 185-206, 2001.

GUIMARÃES, T. Espécie invasora ataca áreas protegidas. Folha de São Paulo, São Paulo, 16 de maio 2005, Folha Ciência, p. A13, 2005.

ITCG. Instituto de Cartografia do Paraná. Disponível em: $<$ (www.icg.pr.gov.br) $>$ Acesso em: 20 de agosto de 2010.

IURK, M. C. et al. Levantamento florístico de um fragmento de Floresta Ombrófila Mista Aluvial do Rio Iguaçu, Município de Palmeira (PR). Floresta, Curitiba, v. 39, n. 3, p. 605-617, jul./set. 2009.

KOZERA, C. et al. Fitossociologia do Componente arbóreo de um fragmento da Floresta Ombrófila Mista Montana, Curitiba, Pr, BR. Floresta, Curitiba, v. 36, n. 2, p. 225-237, maio/ago. 2006.

KUHN, I.; KLOTZ, S. Urbanization and homogenization-Comparing the floras of urban and rural areas in Germany. Biological Conservation, v. 27, p.292-230, 2006.

MAACK, R. Geografia física do Estado do Paraná, 2. ed. J. Olympio, Rio de Janeiro, RJ. 1981, $450 \mathrm{p}$.

MAGGIONI, C.; LAROCCA, J. Levantamento Florístico de um Fragmento de Floresta Ombrófila Mista em Farroupilha/RS. In: X SALÃO DE INICIAÇÃO CIENTÍFICA, 10., 2009, São Leopoldo. Vale do Rio dos Sinos - PUCRS. 2009, p. 185-196,.

MAGURRAN, A. Ecological diversity and its measuement. Princetown: Princetown University Press. 1988, 179 p.

MIELKE, E. et al. Invasive exotic trees in the Conservation Units in Curitiba, Brazil. Acta
Horticulturae, v. 881, p. 493-497, 2010.

MISSOURI BOTANICAL GARDEN. Disponível em: $<$ (www.tropicos.org) $>$ Acesso em: 20 de março de 2010.

MATTEUCCI, S. D.; COLMA, A. Metodologia para el estudo de la vegetacion. Washington:OEA/ PRDECT. 1982, 168 p.

MUELLER-DOMBOIS, D.; ELLEMBERG， H. Aims and methods of vegetation ecology. New York: John Wiley and Sons, 1974, 547 p.

MULLETT T. L. Some characteristics of a native environmental weed: Pittosporum undulatum. In: AUSTRALIAN WEEDS CONFERENCE, 12. 1999, Victoria. Proceedings ... p.592-595, 1999. Tasmanian Weed Society, Devonport. Disponível em: $<$ (www.caws.org.au/awc/1996/awc199614891. pdf) $>$ Acesso em: 08 de maio de 2011.

NEGRELLE, R. R. B.; SILVA, F. C. Fitossociologia de um trecho de Floresta com Araucária Angustifolia (Bert.) O. Ktze. no Município de Caçador - SC. Boletim Pesquisa Florestal, Colombo, n. 25, p. 3754, 1992.

NEGRELLE, R. R. B.; LEUCHTENBERGER, R. Composição e estrutura do componente arbóreo de um remanescente de Floresta Ombrófila Mista. Floresta, Curitiba, v. 1/2, n. 31, p. 42-51, 2001.

OLIVEIRA, A. E. S.; PEREIRA, D. G. Erradicação de espécies exóticas invasoras: múltiplas visões da realidade brasileira Desenvolvimento e Meio Ambiente, n. 21, p. 173-181, jan./jun. 2010.

PIMENTEL A. et al. Fitossociologia do sub-bosque do parque ambiental Rubens Dallegrave, Irati, Pr. Floresta, Curitiba, v. 38, n. 3, jul./set. 2008.

REASER, J. K. et al. Ecological and socioeconomic impacts of invasive alien species in island ecosystems. Environmental Conservation, v. 34, n. 2, p. 98-111, 2007.

REGINATO, M. et al. A vegetação da Reserva Mata Nativa, Curitiba,Paraná, Brasil. Acta Biol. Par., Curitiba, n. 37, p. 229-352, 2008.

RONDON NETO, R. M. et al.. Análise florística e estrutural de um fragmento de floresta ombrófila mista montana, situado em Criúva, RS - Brasil. Ciência Florestal, Santa Maria, v. 12 ， n. 1, p. 29-37, 2002.

ROSE S. Influence of suburban edges on invasion of Pittosporum undulatum into the bushland of northern Sydney, Australia. Australian Journal of Ecology, v. 22, p. 89-99, 1997.

ROSEIRA, D. S. Composição Florística e Estrutura Fitossocioógica do Bosque com Araucária angustifolia (Bert.) O. Ktze no Parque 
Estadual João Paulo II, Curitiba, Paraná. 1990. 111f. Dissertação (Mestrado) Universidade Federal do Paraná, Curitiba, 1990.

ROTTA. E. Identificação dendrológica do parque da Barreirinha, Curitiba-PR. 1977. $257 \mathrm{f}$. Dissertação (Mestrado) - Universidade Federal do Paraná, Curitiba, 1977.

SANQUETTA, C. R. et al. Estabelecimento de plântulas de espécies arbóreas em um experimento de controle de taquaras (Bambusoideae) no sul do Paraná, Brasil. Curitiba: Floresta, v. 35, n. 1, jan./abr. 2005.

SANQUETTA, C. R. Fragmentação da Floresta Ombrófila Mista no Paraná. In: I Simpósio sobre a Mata Atlântica: Conservação, Recuperação e Desenvolvimento. Viçosa: CBCN - Centro Brasileiro para Conservação da Natureza e Desenvolvimento Sustentável, 2005.1 CD-ROM. SCHAAF, L. B. et al. Modificações florístico- estruturais de um remanescente de Floresta Ombrófila Mista Montana no período de 1979 e 2000. Ciência Florestal, Santa Maria, v. 16, n. 3, p. 271-291, 2006.

SESSEGOLO, G. C. A Recuperação de Áreas Degradadas em Unidades de Conservação. In: IAP Unidades de Conservação - ações para valoração da biodiversidade. 2006, p. 25-33.

SNUC - Sistema Nacional de Unidades de Conservação da Natureza. Lei Federal n⿳99985 de 18 de julho de 2000. Disponível em: $<$ (www.planalto. gov.br/ccivil_03/LEIS/L9985.htm)> Acesso em: 08 de maio de 2011.

ZILLER, S. R. A Estepe Gramíneo-Lenhosa no segundo planalto do Paraná: diagnóstico ambiental com enfoque à contaminação biológica. 2000. 268 f. (Doutorado em Engenharia Florestal) Setor de Ciências Agrárias - Universidade Federal do Paraná, Curitiba, 2000. 